\title{
Refeeding Syndrome with Hypoglycemia in a Severely Malnourished Infant
}

\author{
Daiji Takajo $^{1}$ (D) Natalie Sabzghabaei ${ }^{2}$
}

Received: 30 October 2019 / Accepted: 24 December 2019 / Published online: 27 January 2020

(C) Dr. K C Chaudhuri Foundation 2020

\begin{abstract}
A 5-mo-old severely malnourished $3.5 \mathrm{~kg}$ boy was brought to the emergency department with hypoglycemia, bradycardia, bradypnea, and hypothermia. His findings were likely due to severe malnutrition secondary to parental neglect. Resuscitation with dextrose containing intravenous fluids was promptly started. On day 2 of admission, refeeding was initiated. From that time, he had multiple hypoglycemic episodes along with hypophosphatemia, hypomagnesemia, and hypokalemia. Hypoglycemia was associated with the initiation of enteral feeding and an increase in calories and amounts of enteral feeding. Hypoglycemia associated with refeeding syndrome in infant has not been previously reported.
\end{abstract}

Keywords Refeeding syndrome $\cdot$ Severe malnutrition $\cdot$ Infant $\cdot$ Hypoglycemia

\section{Introduction}

Refeeding syndrome is defined as the potentially fatal shifts in fluids and electrolytes that may occur in malnourished patients receiving artificial refeeding [1]. It is caused by increased serum insulin level with refeeding followed by an intracellular shift of glucose, potassium, magnesium, and phosphate [2]. Clinical manifestations usually include hypophosphatemia, hypokalemia, hyperglycemia, and thiamine deficiency [3]. Hyperglycemia is a common associated finding and is explained by insufficient insulin supply at initial feeding [4]. Postparandial hypoglycemia associated with refeeding is reported in some cases with eating disorders [5]. However, hypoglycemia has not been previously reported in infants.

Daiji Takajo

dt1449@gmail.com

1 Department of Pediatrics, Children's Hospital of Michigan, Detroit, MI, USA

2 Department of Hospital Medicine, Carman and Ann Adams Department of Pediatrics, Children's Hospital of Michigan, Central Michigan University College of Medicine, Mount Pleasant, MI, USA

\section{Case Report}

A 5-mo-old boy presents to the emergency department after being found unresponsive by the family. When EMS arrived, the patient was found to have bradycardia, bradypnea with desaturation, hypothermia, and hypoglycemia with a blood sugar undetectable by glucometer. The patient was intubated, warmed, given atropine, and started on intravenous fluids with glucose immediately. On arrival to the hospital, blood pressure was $81 / 45 \mathrm{mmHg}$, heart rate was 110 beats per min, temperature was 36.6 while on warmer, and $\mathrm{SpO}_{2}$ was $100 \%$ on ventilator with $\mathrm{FiO}_{2}$ 0.4. On physical exam, he was severely malnourished with height and weight of $58 \mathrm{~cm}(<1$ percentile for his age) and $3.5 \mathrm{~kg}(<1$ percentile for his age), respectively. Laboratories were sent for genetic, metabolic, and endocrine disorders, which were all unremarkable; no hyperinsulinism in critical samples, no urinary ketones, normal lactate levels, normal serum uric acid level, and normal lipid profile. Patient's severe malnourishment was likely due to inadequate oral intake secondary to parental neglect.

On day 2 of admission, nasogastric feeding was started with $20 \mathrm{kcal} / 30 \mathrm{ml}$ amino acid formula at a rate of $5 \mathrm{ml} / \mathrm{h}$ continuous for $24 \mathrm{~h}(80 \mathrm{kcal} / \mathrm{d})$. Initially, his estimated energy needs for catch up were calculated as $178 \mathrm{kcal} / \mathrm{kg} / \mathrm{d}$ and $3.3 \mathrm{~g} / \mathrm{kg} / \mathrm{d}$ of protein, using his current weight of $3.5 \mathrm{~kg}$ and 


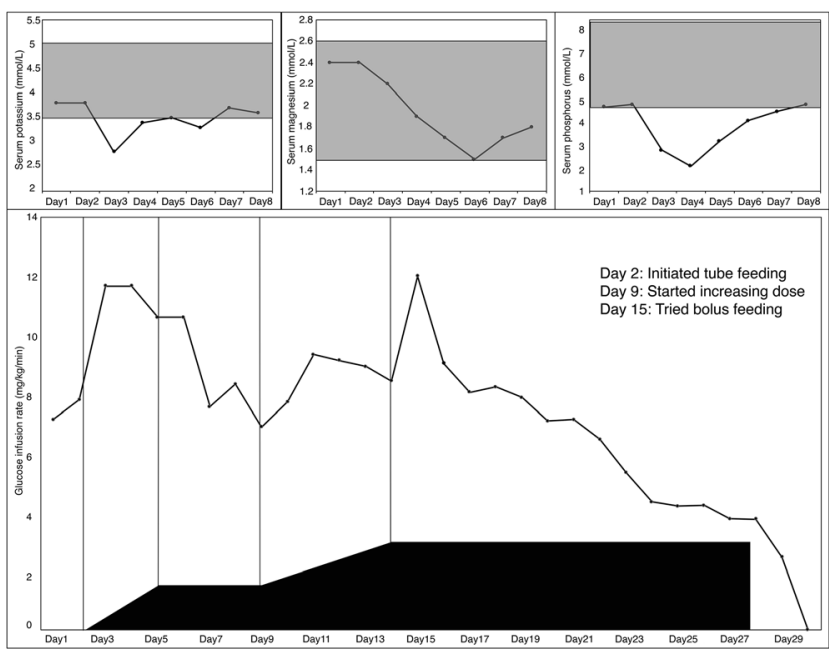

Fig. 1 The graph of index patient showing (a) serum potassium, (b) magnesium, and (c) phosphorus levels. Areas in gray show reference ranges for each electrolyte. (d) shows the trend of glucose infusion rate $(\mathrm{mg} / \mathrm{kg} / \mathrm{min})$ and tube feeding regimen. Shape filled with black shows initiation of tube feeding on day 2 , an increase in dose on day 9 , and reached full feeding on day 15 . Of note, the patient was tried bolus feeding which led to hypoglycemia

ideal body weight for his age of $7.6 \mathrm{~kg}$. His feeding was started with $13 \%$ of his estimated energy needs and was increased by $10 \%$ each day. On day 3 of admission, his serum levels of potassium, magnesium, and phosphorus started to decrease gradually (Fig. 1a-c). Depleted electrolytes were replaced as needed. In addition, IV fluids were started with glucose infusion rate (GIR) of 7.1 on day 1 of admission for hypoglycemia but needed to be increased to 11.7 for multiple hypoglycemic episodes on day 3 of admission. Higher GIR through IV was required to maintain serum blood sugar level when enteral feeding was initiated and when the calories and amounts of enteral feeding were increased. During the period when feeding regimen was unchanged, GIR requirement decreased. On day 15 , continuous tube feeding was switched to bolus feeding which again caused hypoglycemic episodes (Fig. 1d). IV glucose infusion was safely weaned and discontinued on day 30 of admission.

\section{Discussion}

The search of the literature by authors yielded no prior cases of refeeding syndrome with hypoglycemia in infants. In this report, hypoglycemic episodes and higher demand for GIR are associated with enteral refeeding and an increase in the feeding regimen. Hypoglycemia due to refeeding syndrome is potentially caused by increased feeding amounts and calories.

There are no guidelines for refeeding among pediatric populations, although most experts agree that initial refeeding must be slow. Recommendations vary widely, but starting at $20-75 \%$ of estimated caloric needs is prudent [4]. This patient started at $13 \%$ of his estimated energy needs on day 2 of admission and electrolyte disturbances occurred on day 3. Additionally, on day 3 , multiple hypoglycemic episodes occurred with electrolyte disturbances which required electrolyte correction, several IV glucose boluses, and increased glucose infusion rate. The initiation of enteral feeding with a lower dose than $20-75 \%$ of estimated caloric needs could not prevent refeeding syndrome in this case.

Hypoglycemia due to refeeding syndrome has not been frequently reported even in adult patients. Postparandial hypoglycemia in patients with eating disorders has been reported in some adults or adolescent cases [6]. Hypoglycemia is explained by depleted hepatic glycogen reserves and gluconeogenesis substrates. Large glucose loads stimulate substantial amounts of insulin release from the pancreas, which cannot be offset by the depleted hepatic reserves of glycogen [5].

The patient's hypoglycemic episodes can be explained by the same mechanism. Hypoglycemia clearly correlates with enteral refeeding and an increase in the feeding regimen. Trial of bolus feedings on day 15 caused higher insulin surge, which resulted in hypoglycemic episodes and particularly higher GIR requirement.

Hypoglycemia can be one of the manifestations in severely malnourished infants with refeeding syndrome. Extra caution is required when patients initiate enteral refeeding and increase the feed volume.

Acknowledgements The authors thank Department of Pediatric Intensive Care, Genetics, Gastroenterology, Endocrinology, and General Pediatrics at Children's Hospital of Michigan for patient care.

Authors' Contribution DT, NS: patient care; DT: writing the manuscript; all authors: critically reviewing the manuscript. NS will act as guarantor for this paper.

\section{Compliance with Ethical Standards}

Conflict of Interest None.

\section{References}

1. Mehanna HM, Moledina J, Travis J. Refeeding syndrome: what it is, and how to prevent and treat it. BMJ. 2008;336:1495-8.

2. Crook M, Hally V, Panteli J. The importance of the refeeding syndrome. Nutrition [Internet]. 2001;17:632-7.

3. Kliegman RM, Stanton BMD, St. Geme J, Schor NF. Nelson Textbook of Pediatrics, 20th ed. Philadelphia: Elsevier; 2016.

4. Pulcini CD, Zettle S, Srinath A. Refeeding syndrome. Pediatr Rev [Internet]. 2016;37:516-23.

5. Mehler PS, Winkelman AB, Andersen DM, Gaudiani JL. Nutritional rehabilitation: practical guidelines for refeeding the anorectic patient. J Nutr Metab. 2010;2010. https://doi.org/10.1155/2010/625782.

6. Hart S, Abraham S, Franklin RC, Twigg SM, Russell J. Hypoglycaemia following a mixed meal in eating disorder patients. Postgrad Med J. 2011;87:405-9.

Publisher's Note Springer Nature remains neutral with regard to jurisdictional claims in published maps and institutional affiliations. 